\title{
The Impact of Non-nominal Yaw Attitudes of GPS Satellites on Kinematic PPP Solutions and their Mitigation Strategies
}

\author{
Yidong Lou, Fu Zheng, Shengfeng Gu and Yang Liu \\ (GNSS Research Center, Wuhan University, Wuhan, China) \\ (E-mail: gsfjay@163.com)
}

\begin{abstract}
The yaw attitudes of Global Positioning System (GPS) satellites are critical to both satellite antenna phase centres and the phase wind-up corrections. However, the nominal yaw attitude of GPS satellites can barely be maintained during eclipse seasons. Thus, several yaw attitude models have been developed by the International GNSS Service Analysis Centres (IGS ACs) to avoid positioning degradation caused by non-nominal yaw attitudes. Based on the analysis of the strategy of each AC, the modelled yaw attitude is compared with the nominal one, and the residuals of Precise Point Positioning (PPP) are investigated to evaluate the influence of non-nominal attitudes with over one year of observable data collected from 15 IGS stations. The results suggest that non-nominal attitudes of BLOCK II/IIA satellites have the largest impact of about $20-30 \mathrm{~cm}$, and their positioning accuracy can be improved by $20 \%-30 \%$ with the corresponding yaw attitude model. Similar results have also been demonstrated with BLOCK IIR and BLOCK IIF satellites. Furthermore, compared with the strategy of directly deleting the data for the eclipsing seasons, PPP with the yaw-attitude model achieves better results by about $30 \%$ when the satellite geometry is relatively poor.
\end{abstract}

\section{KEY WORDS \\ $\begin{array}{lll}\text { 1. GPS Satellite Eclipsing. 2. Yaw Attitude. 3. Kinematic PPP } & \text { 2. }\end{array}$}

Submitted: 2 September 2014. Accepted: 11 January 2015. First published online: 12 February 2015.

1. INTRODUCTION. In recent years, Precise Point Positioning (PPP) has become a powerful tool in estimating station positions with an accuracy of millimetre to decimetre-level in both static and kinematic applications (Zumberge et al., 1997; Kouba and Heroux, 2001; Bisnath and Gao, 2008). To achieve the highest positioning accuracy, users should implement the same conventions and models as those adopted by the International GNSS Services (IGS) (Dow et al., 2009), such as solid earth tides, phase wind-up (Wu et al., 1993), satellite antenna offset, etc. (Kouba, 2009a), as well as the yaw attitudes of Global Positioning System (GPS) satellites which relate critically to the solar radiation pressure model, the corrections of satellite antenna phase centre offset and phase wind-up. At present, however, IGS Analysis Centres 
(ACs) adopt mixed models for GPS attitudes, which will confuse PPP software developers and lead to relatively poor positioning results.

During satellite eclipsing periods, how to accurately model the non-nominal yaw attitudes or eliminate the effect of non-nominal yaw attitudes is of particular interest to the GPS community. Bar-Sever (1996) introduced the yaw attitude modelGYM95 (GPS Yaw attitude Model-95) to determine the non-nominal attitude and its durations for BLOCK IIA satellites during the noon turn and shadow crossing. For BLOCK IIR satellites, an additional model was developed by Kouba (2009b) based on the simplification of the GYM95. Furthermore, Dilssner (2010) analysed the yaw attitudes of the BLOCK IIF satellites with concentration on the antenna phase centre offset and its variation.

Though the efficiency of the above models has already been confirmed in various studies, there is still no consensus on the strategy of treating an eclipsing satellite among IGS ACs. Jet Propulsion Laboratory (JPL) and EMR (Geodetic Survey Division of Natural Resources Canada (NRcan), formerly Energy, Mines and Resources) apply the GPS satellite yaw attitude model and estimate yaw rates as well and delete the 30 minute observations during the post-shadow recovery period; the Massachusetts Institute of Technology (MIT) applies GYM-95 based on nominal yaw rates and retains the observation after a BLOCK II/IIA satellite exits eclipse shadow (Bar-Sever, 1996); Similar to MIT, Geoforschungszentrum (GFZ) applies the nominal yaw rates to determine the non-nominal yaw attitude of satellites, but deletes the 30 minutes observations after a BLOCK II/IIA satellite exits eclipse shadow (Bar-Sever, 1996). GFZ also estimates the yaw rates of the BLOCK II/IIA and BLOCK IIF satellites during shadow crossing; the European Space Agency (ESA) implements the nominal yaw attitude without considering the eclipsing seasons; GRG (Groupe de Recherche de Geodesie Spatitle (space geodesy research group)-Centre National d'Etudes Spatiales (CNES) / Collecte Localisation Satellites(CLS)) applies Kouba (2009b) yaw attitude models for eclipse seasons and retained the observations of the BLOCK II/IIA satellites during post-shadow recovery (ftp://igscb.jpl.nasa.gov/igscb/center/analysis). Regarding the studies above, it is concluded that the yaw attitude recovery of BLOCK IIA satellite from shadow exit takes about 30 minutes and most of IGS ACs simply reject the measurements from this period.

Consequently, on the one hand, various strategies in non-nominal yaw attitude treatment of individual AC may lead to inconsistency in PPP applications since the users do not know exactly how these orbit and clock products are generated; on the other hand, considering urban applications with poor satellite geometry, deleting the eclipsing satellite will cause unexpected positioning errors in PPP. Thus in this contribution, we focused on the analysis of the non-nominal yaw attitude for different GPS satellite types and its impact on PPP.

This paper is organised as follows: Section 2 introduces the GPS satellite attitude and the strategies to mitigate the impact of non-nominal yaw attitude. The data and processing strategy are shown in Section 3. By the comparison between the nominal and the modelled yaw angle during eclipsing seasons, as well as the analysis of residuals distribution, Section 4 presents the impacts of non-nominal yaw attitudes on PPP and demonstrates the improvement in the positioning accuracy through the application of our proposed processing strategy. Finally, Section 5 summarises our conclusions and suggestions. 


\section{SATELLITES YAW ATTITUDE AND STRATEGIES OF MITIGATING NON-NOMINAL ATTITUDES}

2.1. Satellite Attitude. The yaw attitude of GPS satellites can be defined in a satellite-based coordinate system with the Z-axis pointing to the centre of the Earth, the Y-axis pointing along the solar panels and perpendicular to the direction of the satellite-Sun, the X-axis completing the orthogonal frame and pointing toward the Sun for BLOCK II/IIA and BLOCK IIF or away from the Sun for BLOCK IIR satellites (Kouba, 2009b; Dilssner, 2010; Dilssner et al., 2011). The nominal yaw attitude of a GPS satellite is governed by two constraints: 1) The transmitter antenna always points toward the Earth; 2) The solar panel is always perpendicular to the SatelliteSun direction.

Due to the constraints of satellite payload and orbit design, non-nominal yaw attitudes of a GPS satellite can be divided into three categories:

1) When satellites are at the noon where $\mu=180^{\circ}$ ( $\mu$ is the geocentric orbital angle between the satellite and the orbit midnight, growing with the satellite motion), they need a rapid rotation to keep the X-axis pointing toward or away from the Sun. However, the maximum yaw speed cannot maintain this rapid rotation and thus non-nominal yaw attitude occurs (this phase is called the noon turn);

2) When the Sun, satellite, and the Earth are collinear and satellite is in eclipse, the $\mathrm{X}$-axis no longer keeps pointing toward the Sun, this phase is called the shadow crossing (the mechanism in BLOCK IIR satellites during shadow crossings is similar to the noon turn, and thus it is known as the night turn) (Kouba 2009b);

3) At the shadow-exit moment, the BLOCK II/IIA and BLOCK IIF satellites cannot recover the normal yaw attitude immediately. Thus, this phase is called the post-shadow recovery (Bar-Sever, 1996; Kouba, 2009b). In the following two subsections, we introduce two approaches for dealing with non-nominal satellite attitudes during eclipsing seasons.

2.2. Strategy 1: Deleting observations for Eclipsing Satellites. Awell-known strategy to mitigate non-nominal attitudes is to remove the measurements of the eclipsing satellites. Generally, there are still enough satellites after such observations are removed, and PPP users can still obtain high positioning accuracy. However, for mobile users, the increasing unknowns and the weak constraints on positioning among adjacent epochs always make data deletions unfavourable. Ambiguity initialisation and slow convergence can degrade the accuracy of PPP solutions, especially when a few satellites undergo the eclipsing period simultaneously (Kouba, 2009b).

2.3. Strategy 2: Modelling Non-nominal Yaw Attitudes for Eclipsing Satellites. Usually the yaw attitudes of satellites are determined by their yaw angles. When the yaw attitude of a satellite is normal, the yaw angle and the satellite-based X-axis orientation satisfy:

$$
\varphi=\operatorname{arcos}(\mathrm{v} \cdot \mathrm{x})
$$

where $\mathrm{v}$ is the inertial satellite velocity unit vector and $\mathrm{x}$ is the satellite-based $\mathrm{X}$-axis orientation unit vector in the inertial frame (Kouba, 2009b).

When the yaw attitude of satellites is non-nominal, we apply the nominal yaw rates to correct the yaw attitude (Bar-Sever, 1996; Kouba, 2009b; Dilssner et al., 2011). The 
Table 1. Non-nominal yaw attitude characteristics of different satellite types.

\begin{tabular}{llccc}
\hline Satellite types & $\begin{array}{l}\text { Nominal yaw } \\
\text { rate/(\%/s) }\end{array}$ & $\begin{array}{l}\text { Noon } \\
\text { turn/(min) }\end{array}$ & $\begin{array}{l}\text { Shadow } \\
\text { crossing/(min) }\end{array}$ & $\begin{array}{l}\text { Shadow } \\
\text { recovery } /(\min )\end{array}$ \\
\hline BLOCK II/IIA & $0 \cdot 11-0 \cdot 13$ & $<30$ & $\sim 60$ & $<30$ \\
BLOCK IIR & $0 \cdot 2$ & $<15$ & $\sim 15$ & 0 \\
BLOCK IIF & $0 \cdot 06 / 0 \cdot 11(\max )$ & $<30$ & $\sim 55$ & $<10$ \\
\hline
\end{tabular}

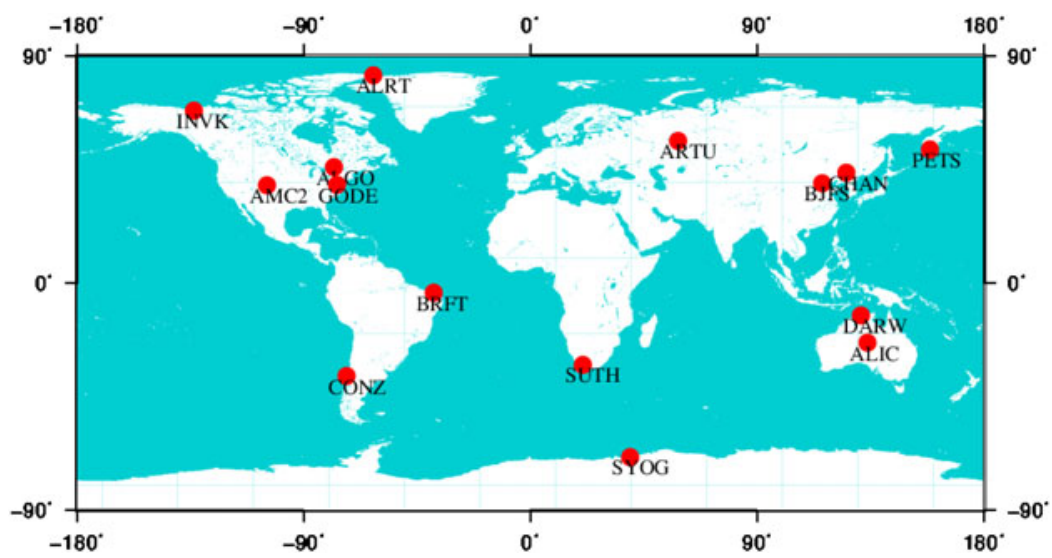

Figure 1. Tracking stations chosen from the IGS network.

specific expression of yaw angle for BLOCK II/IIA and BLOCK IIF satellites is

$$
\varphi(t)=\operatorname{atan} 2(-\tan \beta, \sin \mu)+\operatorname{SIGN}\left(\omega_{\varphi}, \beta\right) \cdot\left(t-t_{s}\right)
$$

Since the X-axis orientation of BLOCK IIR satellites is away from the Sun, the calculation equation for BLOCK IIR satellites is

$$
\varphi(t)=\operatorname{atan} 2(\tan \beta,-\sin \mu)+\operatorname{SIGN}\left(\omega_{\varphi}, \beta\right) \cdot\left(t-t_{s}\right)
$$

In practical applications, the yaw angle of BLOCK II/IIA and BLOCK IIF satellites during the post-shadow period is

$$
\varphi(t)=\varphi\left(t_{e}\right)+\operatorname{SIGN}\left(\omega_{\varphi}, \Delta\right) \cdot\left(t-t_{e}\right)
$$

where $\mathrm{t}$ is the current epoch time, $t_{s}$ is the start time of the non-nominal yaw attitude and $t_{e}$ is the time for shadow exit (Kouba, 2009b); $\beta$ is the elevation of the Sun with respect to orbital plane, SIGN is the usual FORTRAN sign function, $\omega_{\varphi}$ is the yaw rate of satellites and its nominal value is shown in Table 1. $\Delta$ is the difference between the modelled yaw angle and the nominal yaw angle at $t_{e}$.

3. DATA. For in-depth study of the non-nominal yaw attitudes and their impact on the PPP solutions, we selected 15 IGS tracking stations and 30-second interval data from DOY 153 of 2012 to DOY 151 of 2013. The distribution of the stations is shown in Figure 1. As Figure 1 shows, the selected stations are distributed worldwide, 

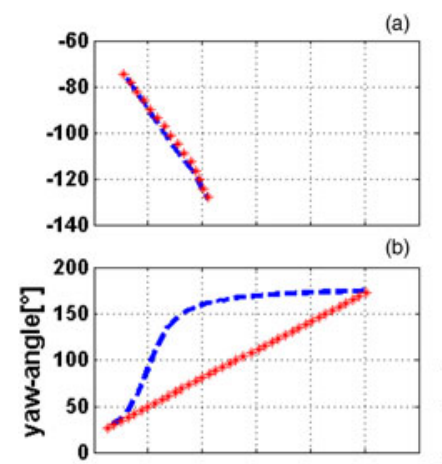

(c)
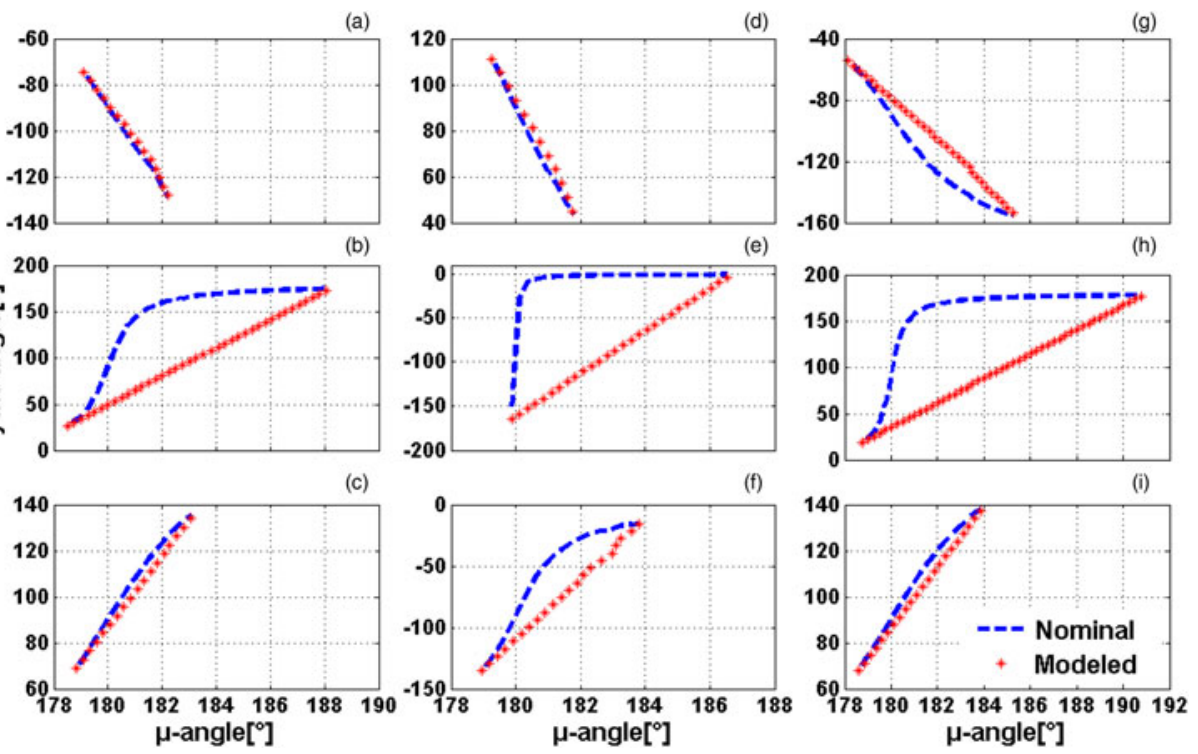

Figure 2. Comparison of yaw angle of BLOCK IIA (G09, left column), IIR (G11, middle column) and IIF (G01, right column) during noon turn, the $\beta$ for every subfigure is shown in Table 2.

with about one year of these data, we can also analyse the impact of non-nominal yaw attitude on stations in different areas.

Orbits and 30-second clocks from IGS and individual ACs are used for analysis of the non-nominal yaw attitude during the kinematic PPP processing. The tropospheric delays are calculated with the Saastamoinen model (Saastamoinen, 1971) and Niell mapping function (Niell, 1996) using predicted meteorological parameters, while the remaining are estimated as a random walk process. Receiver clocks are estimated epoch-by-epoch, station coordinates are estimated epoch-by-epoch and no integer ambiguity fixing is done. New ambiguity is inserted once a cycle slip is detected and observations identified as outliers are down-weighted (Liu and Ge, 2003; Shi et al., 2008; Geng et al., 2010; 2011). We adopt the yaw-attitude model (Kouba, 2009b) in the Positioning and Navigation Data Analyst (PANDA) software and compare the two different mitigation strategies from Section 2.

\section{RESULTS AND DISCUSSION}

4.1. Difference between Nominal and Modelled Yaw Angle. When the GPS satellites are eclipsing, the modelled yaw angle of different satellite types can be calculated with Equations (2), (3) and (4) whereas the nominal yaw angles are calculated with Equation (1). Then we compare the differences between the nominal and modelled yaw angles of different GPS satellite types at the noon turn, during shadow crossing and post-shadow recovery periods, respectively. Additionally, we analyse the duration of the non-nominal yaw attitude for different satellite types.

4.1.1. Yaw Angles at Noon Turn. Figure 2 shows the variation of the nominal and the modelled yaw angles of BLOCK IIA, IIR and BLOCK IIF satellites during noon 
Table 2. $\beta$ for every subfigure of Figure 2 .

\begin{tabular}{ccc}
\hline BLOCK IIA G09 & BLOCK IIR G11 & BLOCK IIF G01 \\
\hline $3 \cdot 27^{\circ}(\mathrm{a})$ & $1 \cdot 91^{\circ}(\mathrm{d})$ & $2 \cdot 61^{\circ}(\mathrm{g})$ \\
$-0 \cdot 76^{\circ}(\mathrm{b})$ & $-0 \cdot 06^{\circ}(\mathrm{e})$ & $-0 \cdot 41^{\circ}(\mathrm{h})$ \\
$-3 \cdot 05^{\circ}(\mathrm{c})$ & $-1 \cdot 04^{\circ}(\mathrm{f})$ & $-3 \cdot 45^{\circ}(\mathrm{i})$ \\
\hline
\end{tabular}

(a)
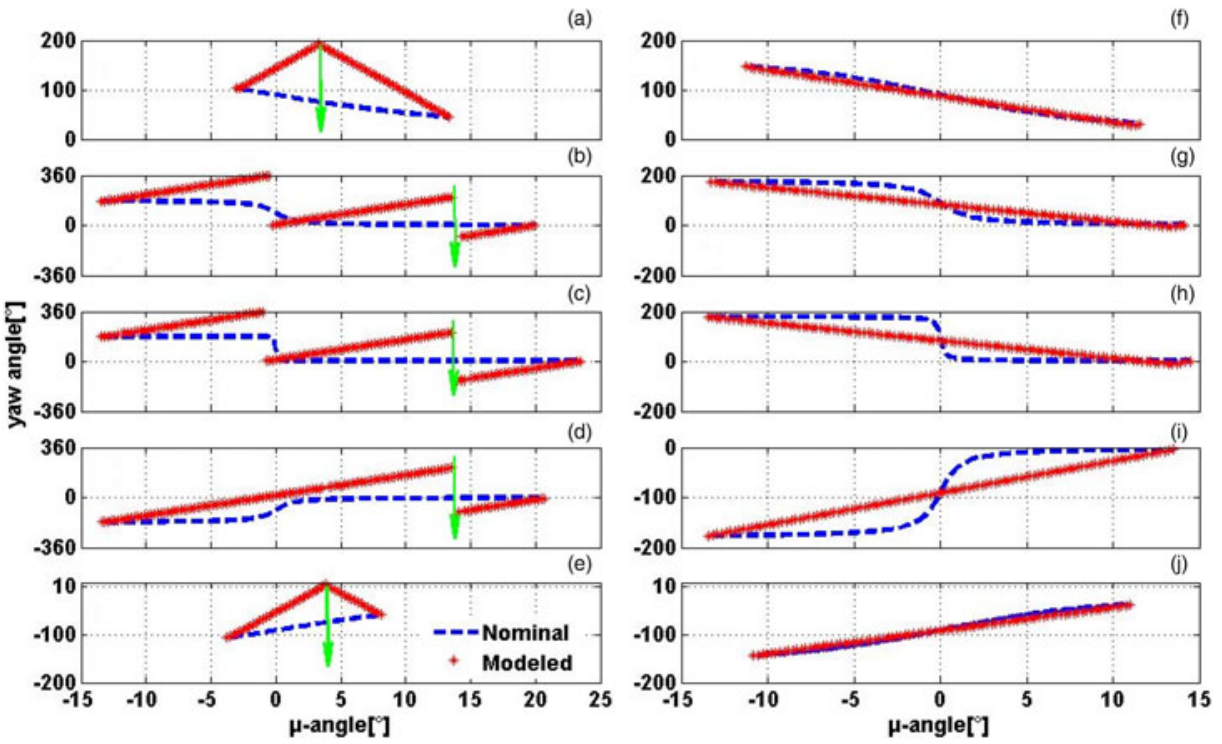

Figure 3. A comparison of yaw angle of a BLOCK IIA satellite (G04, left column, the green arrow denotes the period after shadow exit point and the periods before green arrows are shadow crossing) and IIF (G01, right column) during shadow crossing and post-shadow recovery, the $\beta$ for every subfigure is shown in Table 3.

turn. The differences between the nominal and the modelled yaw angles in this period are related to the Sun elevation angles regardless of the satellite type. As shown in subfigures $b$, e and $h$ of Figure 2, the Sun elevation angle is approximately $0^{\circ}$ and the differences between the nominal yaw angle and the modelled yaw angle can be up to around $100^{\circ}$. When the Sun elevation angle is larger, as shown in sub-figures a, c, d and $i$ of Figure 2, the differences between the nominal and the modelled yaw angle become smaller and the duration of the noon turn is hence short.

4.1.2. Yaw Angles during Shadow Crossing and Shadow Recovery. As the regime of shadow crossing of BLOCK IIR is the same as that at noon turn (Kouba, 2009b), we only compare the yaw angles of BLOCK IIA and BLOCK IIF. Figure 3 shows the differences between the nominal and the modelled yaw angles for BLOCK IIA and BLOCK IIF during shadow crossing and shadow recovery. As shown in Figure 3, the Sun elevation angle has a different impact on the yaw angle of BLOCK IIA and BLOCK IIF satellites. The yaw rates of the BLOCK IIA satellites when crossing the shadow are always positive, and are not associated with the Sun elevation angle while the positive or negative yaw rates of a BLOCK IIF satellite relate to the Sun 
Table 3. $\beta$ for every sub-figure of Figure 3.

\begin{tabular}{lrrrrr}
\hline BLOCK IIA G04 & $-13 \cdot 11^{\circ} \mathrm{a}$ & $-1 \cdot 04^{\circ} \mathrm{b}$ & $-0 \cdot 04^{\circ} \mathrm{c}$ & $0 \cdot 97^{\circ} \mathrm{d}$ & $12 \cdot 95^{\circ} \mathrm{e}$ \\
BLOCK IIF G01 & $-7 \cdot 24^{\circ} \mathrm{f}$ & $-1 \cdot 18^{\circ} \mathrm{g}$ & $-0 \cdot 16^{\circ} \mathrm{h}$ & $0.84^{\circ} \mathrm{i}$ & $7 \cdot 87^{\circ} \mathrm{j}$ \\
\hline
\end{tabular}

elevation angle. Since the GPS Control Segment adds a $0 \cdot 5^{\circ}$ yaw bias permanently to BLOCK II/IIA satellites during shadow crossings (Bar-Sever, 1996), the yaw rates of BLOCK II/IIA satellites are always positive during shadow crossing periods. In addition, the differences between the nominal and the modelled yaw angles of BLOCK II/IIA satellites are still large with the increasing of the Sun elevation angle (Figure 3.a and 3.e) while the differences between nominal and modelled yaw angles of BLOCK IIF satellites are small when the Sun elevation angle is large (Figure 3.f and 3.j). Compared to the BLOCK II/IIA satellites, the yaw rates of BLOCK IIF satellites when crossing the shadow are smaller, about $0.06 \%$, and the non-nominal yaw attitude of BLOCK IIF satellites has little impact on the yaw angle i.e. the difference of nominal and real attitude of BLOCK IIF is small during shadow crossing.

Figure 3 also shows the variation of yaw angles after shadow exit. The period of post-shadow recovery of the BLOCK IIA satellite is longer than that of the BLOCK IIF (the period of post-shadow recovery for BLOCK IIF is very short, but is not obvious in Figure 3). This is because the period of post-shadow recovery relates to the difference between the nominal and the real yaw angle at the shadow exit. The maximum yaw rates of satellites, as well as the sign of yaw rates for BLOCK IIF satellites related to the Sun elevation angle, making the differences between the nominal and the modelled yaw angle small. The yaw rate of BLOCK IIF satellites in post-shadow recovery is $0.11 \%$, thus the maximum recovery time should not exceed $10 \mathrm{~min}$ (Dilssner et al., 2011). The yaw rates of BLOCK IIA satellites during shadow crossings are permanently positive and the difference of yaw angles at shadow exit can be up to $100^{\circ}$ or even more (Figure 3.a). So the recovery time of a BLOCK IIA satellite is longer than that of a BLOCK IIF satellite. In addition, the differences between the nominal and the modelled yaw angle may be up to $360^{\circ}$ when a BLOCK IIA satellite is at the shadow exit, as shown in Figure 3.b, 3.c and 3.d. Consequently, when using the nominal yaw attitude, the one-cycle difference will affect the phase wind-up correction for succeeding epochs.

4.2. Impact of Non-nominal Yaw Attitude on PPP and application of Mitigating Strategies. Based on the analysis of non-nominal yaw attitude of different satellite types in Section 4.1, it is concluded that the impact of the non-nominal yaw attitude on BLOCK II/IIA satellites are the largest while the effects on BLOCK IIR and BLOCK IIF satellites are small. We then analyse the impacts of different satellite types on the PPP solution during eclipsing seasons.

4.2.1. Analysis of observation residuals during Eclipsing Seasons. The precise orbit and clock products in PPP processing are usually obtained by a combination of different ACs, but the strategies to mitigate non-nominal yaw attitudes are not the same for different ACs. Thus the clock products may have inconsistencies between 0.5 to $1.5 \mathrm{~ns}$ (Zhang et al., 2010), which cannot be ignored in PPP solutions. We then select the IGS station AMC2 and analyse the observation residuals with different products of ACs and IGS during the periods of non-nominal yaw attitudes. The observation residual is calculated through a static PPP solution, down-weighting 


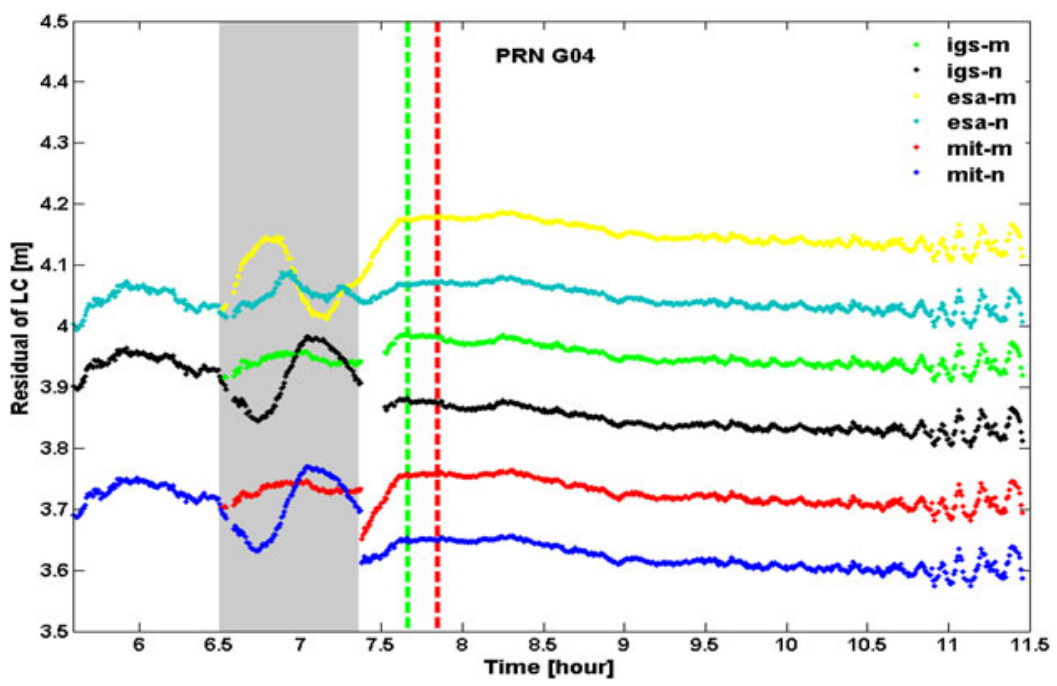

Figure 4. Residual of BLOCK IIA (G04) with nominal and modelled yaw attitudes of different ACs respectively. Shaded bar indicates the eclipsing period, and from the time of shadow exit to the time represented by the green dashed line is the post-shadow recovery period in the yawattitude model, to the time represented by the red dashed line is the constant 30-min postshadow recovery period. The residual is offset by $0.2 \mathrm{~m}$ for ESA and $0.3 \mathrm{~m}$ for MIT to avoid overlapping. The shaded bars and dashed lines for the following figures are the same.

the observations of satellites with non-nominal yaw attitude in the adjustment, i.e. the weight of the corresponding observations effectively to zero. We then directly output the residuals (ambiguity included) of the satellite whose yaw attitude is nonnominal. In order to briefly analyse the impact of different strategies on the observation residual, we only compare ESA (nominal attitude implemented) and MIT (applied yaw attitude model) products.

4.2.1.1. Residuals of BLOCK IIA. Figure 4 shows the ionosphere-free carrierphase residuals for a BLOCK IIA satellite (G04) with different products during DOY 68 of 2013. We find that different strategies in modelling non-nominal yaw attitude have different impacts on the user positioning. When using the nominal yaw attitude, during the period of shadow crossing, the observation residuals with MIT product and IGS final product significantly increase while the residuals with ESA product is consistent with the other periods. This is because the ESA products do not account for the non-nominal yaw attitude, and users can thus obtain high accuracy even if applying the nominal yaw attitudes. Conversely, when using the yaw-attitude model, the residuals with ESA products are worse than that with the MIT products and IGS final products. The strategy of MIT is inconsistent with other ACs during post-shadow recovery period. In particular, they retain measurement during this period without any corrections, and thus the residuals time series with the same kind of jump should be in the satellite clock at the shadow exit. Therefore, users must delete the data during this period when using the products from MIT.

By comparison of the residuals with nominal yaw attitude and the yaw-attitude model, we can clearly see that when using the nominal yaw attitude, the residuals based on MIT or IGS products have about $10 \mathrm{~cm}$ variations which can be reduced 


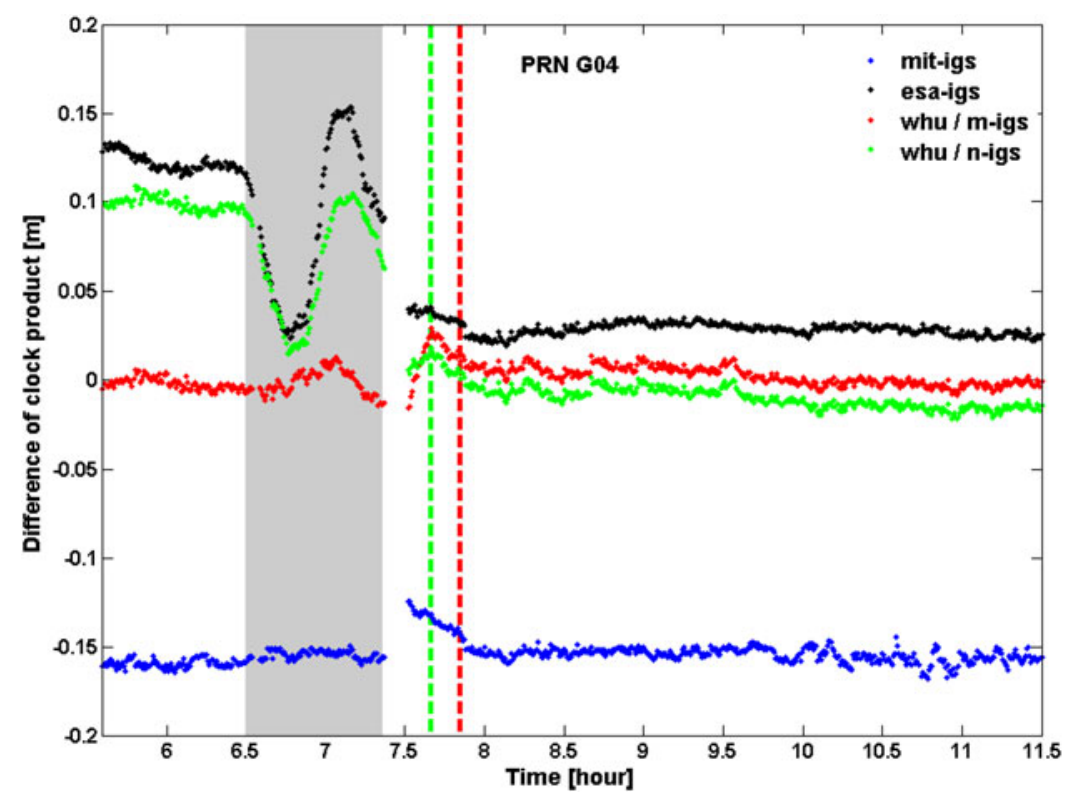

Figure 5. Differences in clock products for different ACs and IGS, whu / $\mathrm{m}$ represents the clock through the yaw-attitude model while whu / $\mathrm{n}$ is the clock estimated with the nominal yaw attitude.

by using the yaw-attitude model. We made a further analysis on the non-nominal yaw attitude corrections for precise products and user positioning using two strategies, i.e. applying the yaw-attitude model and applying the nominal yaw attitude. Figure 5 shows the differences between 30 -second precise clock products from different ACs including PANDA clock product and the IGS final clock product. For the clock products from either ESA or PANDA without consideration of the non-nominal yaw attitude, the clock differences reach about $10 \mathrm{~cm}$ which have the same magnitude in residual as those derived from nominal yaw attitude with MIT products (Figure 4). Thus our research shows that the ESA clock product absorbs parts of the errors resulting from the non-nominal yaw attitude, and the residual from ESA products that applied the nominal yaw attitude is smaller than that applied to the yaw-attitude model. Because the clocks cannot completely absorb the errors resulting from the non-nominal yaw attitude, the residuals resulting from the nominal attitude in the ESA product are slightly larger than those resulting from the yaw-attitude model in the MIT products during the eclipsing period.

This shows that applying the yaw-attitude model can greatly reduce the errors resulting from non-nominal yaw attitude during the shadow crossing period for the BLOCK IIA satellites and repair the one-cycle slip. As shown in Figure 4, the observations residual after repair is consistent with the residual in other periods of normal yaw attitude, but the effect is not satisfactory, which needs further investigation.

4.2.1.2. Residuals of BLOCK IIR and BLOCK IIF. Figures 6 and 7 present the residual time series for the BLOCK IIF satellite G24 and BLOCK IIRM satellite G07 in DOY 55 of 2013. These figures show that the fluctuations resulting from the non-nominal yaw attitudes of the BLOCK IIF or BLOCK IIR are smaller than 


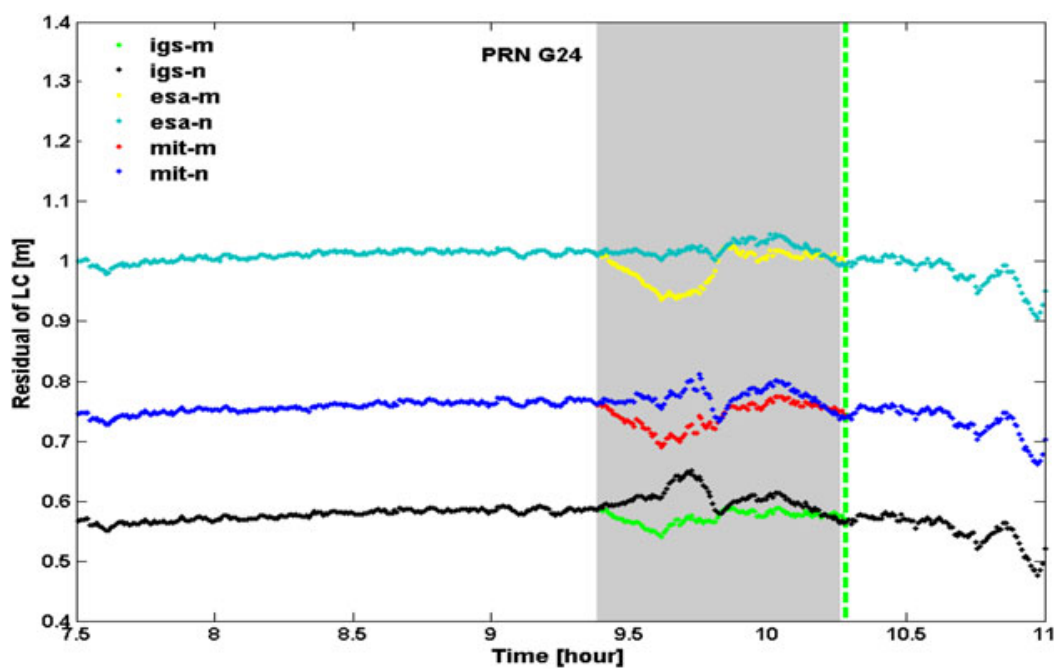

Figure 6. Residual of BLOCK IIF (G24) with nominal and modelled yaw attitudes of different ACs respectively. The residual is offset by $0.2 \mathrm{~m}$ for ESA and $0.3 \mathrm{~m}$ for MIT to avoid overlapping.

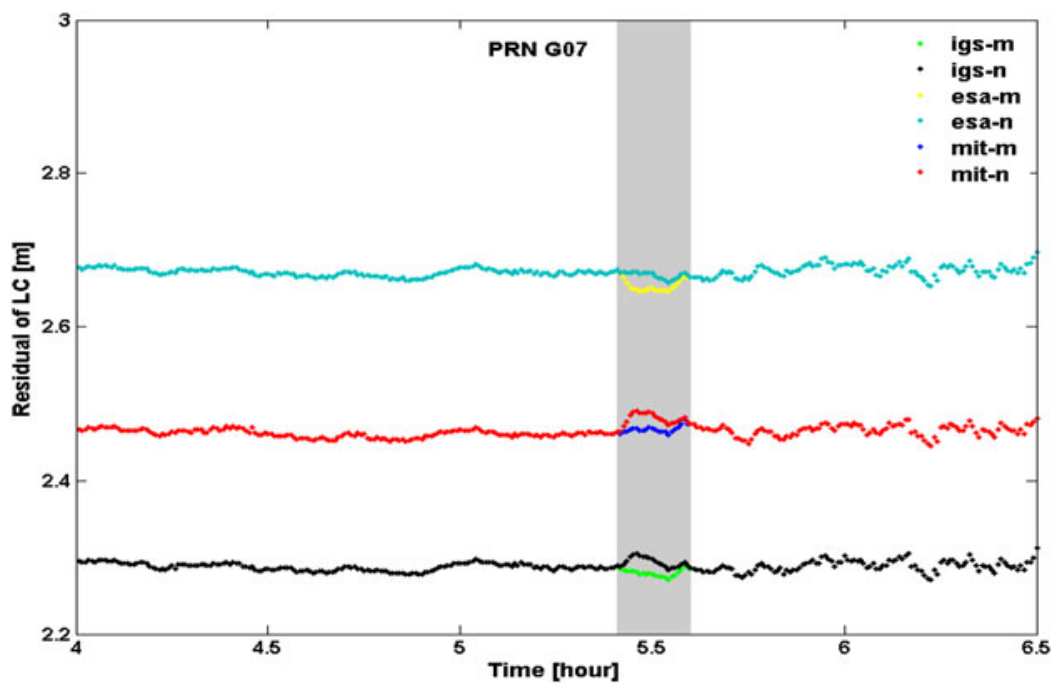

Figure 7. Residual of BLOCK IIRM (G07) with nominal and modelled yaw attitudes of different ACs respectively. The residual is offset by $0.2 \mathrm{~m}$ for ESA and $0.3 \mathrm{~m}$ for MIT to avoid overlapping.

those of the BLOCK IIA satellite. Additionally, the post-shadow recovery period of the BLOCK IIF is shorter than that of the BLOCK IIA satellite, i.e. only a few minutes.

By comparing Figures 4-7, we can conclude that the impact of non-nominal yaw attitude can be reduced by the yaw-attitude model for the products which are corrected for the non-nominal yaw attitude, except for ESA. Although the IGS combined clock 

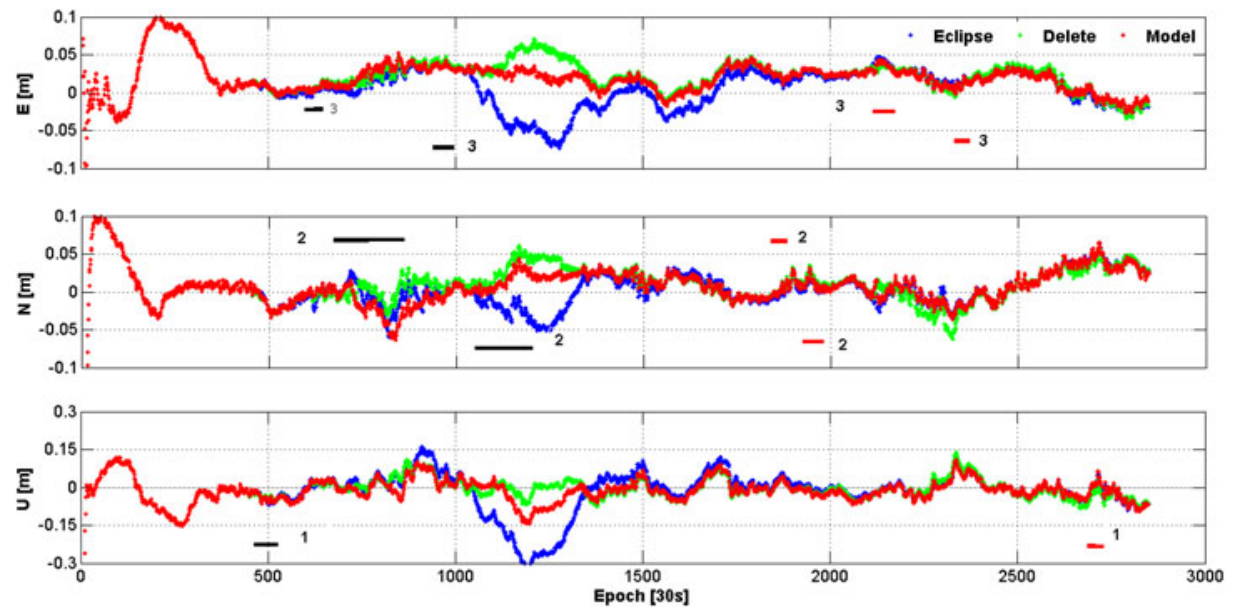

Figure 8. The position differences at AMC2 station for three strategies, 1 3 represent BLOCK IIF, BLOCK IIA and BLOCK IIR in eclipsing seasons, respectively. The black lines represent shadow crossing and red lines represent the noon turn.

for the eclipsing periods is not clearly defined, the residual of IGS final products is consistent with the ACs that take into consideration the non-nominal yaw attitude.

4.2.2. Kinematic PPP Solutions during the periods of Non-nominal Yaw Attitudes. From the above analysis, we know that the impact of non-nominal attitudes of BLOCK IIA on PPP residuals is larger than that of BLOCK IIR and BLOCK IIF. We will now analyse the impact of non-nominal yaw attitudes on kinematic PPP solutions. At first, we compare the PPP solutions under different conditions including poor satellite geometry distribution and then we evaluate the positioning error for different satellite types and compare the different strategies of mitigating non-nominal attitudes to find an optimal strategy to correct for non-nominal yaw attitude.

In this section, three strategies are used for kinematic PPP solutions: 1) Apply the nominal yaw attitude; 2) Delete the data during eclipsing seasons and 3) Apply the yaw-attitude model. In the following figures, strategies 1,2 and 3 are represented as Eclipse, Delete and Model, respectively. We compared the coordinate of every epoch during eclipsing seasons with the coordinates supplied by IGS weekly solutions, and calculated the RMS of their differences.

4.2.2.1. PPP solutions under different conditions. Figure 8 shows the time series of kinematic PPP solutions for station AMC2 where we can observe the eclipsed satellites including one BLOCK IIF, four BLOCK IIA and four BLOCK IIR satellites on DOY 78 of 2013. It is seen that, as concluded in the above section, the impacts of the non-nominal yaw attitude of the BLOCK IIA satellites on the positioning are the largest, especially in the Up direction and its positioning error reaches $20-30 \mathrm{~cm}$, while the impacts of BLOCK IIR and BLOCK IIF satellites on the positioning are not significant. On average, there are nine satellites in view during this day and the satellite geometry is good, so strategies 2 and 3 show similar positioning performance.

Although the positioning accuracy above with strategy 2 is equal to strategy 3 , strategy 2 is at the expense of discarding some observation data. Thus, under the 


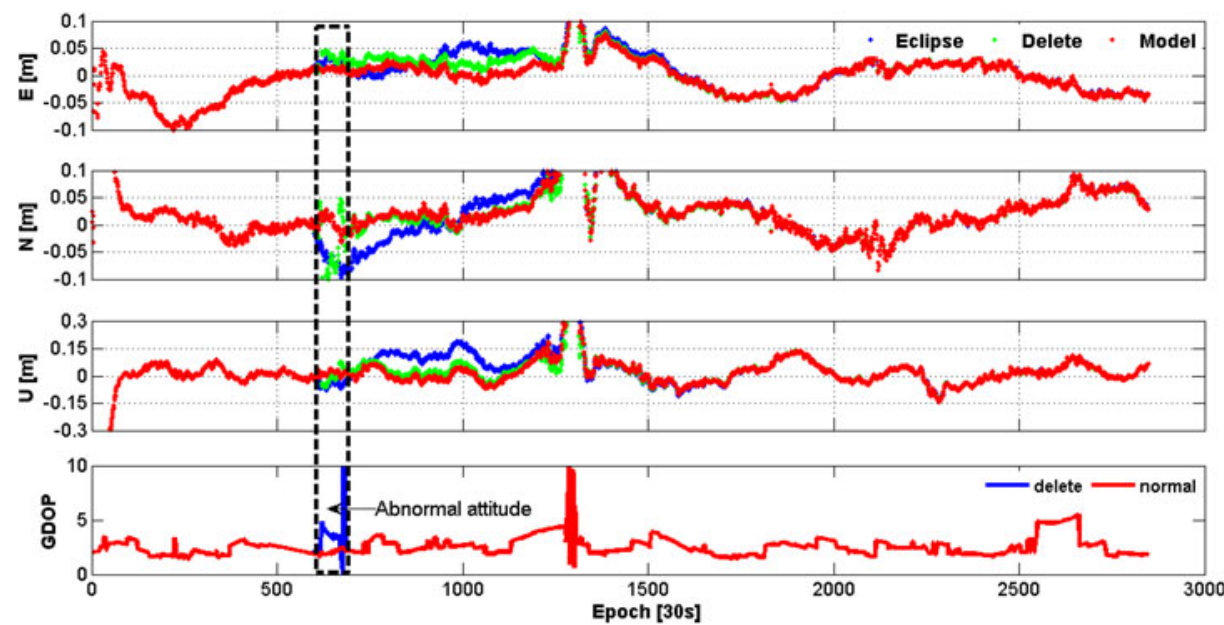

Figure 9. The position differences at AMC2 station for three strategies. The rectangular area represents the eclipsing period.

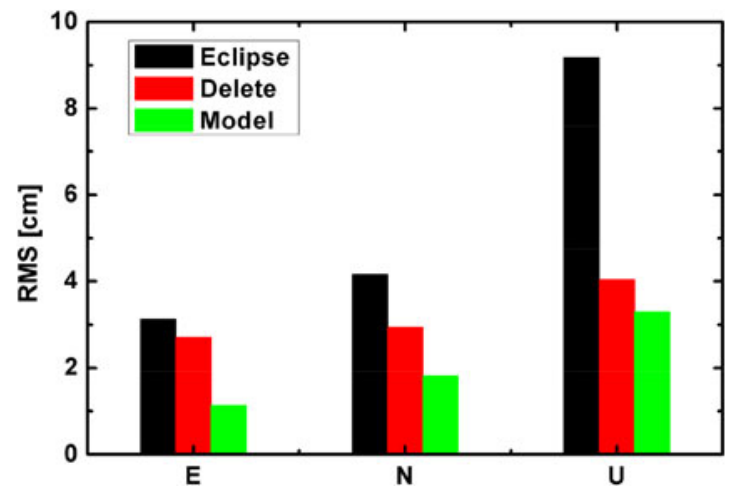

Figure 10. Positioning RMS in the North, East and Up directions at station AMC2 of DOY 81 of 2013.

condition of few redundant observations in this period, strategy 2 will impact the positioning negatively. In the following, we selected the IGS station AMC2 in DOY 81 of 2013, and compared the positioning accuracy of the three strategies by manually deleting the satellites in the same quadrant as G08 (BLOCK IIA) whose yaw attitude is nonnominal. During the shadow crossing period, there are six satellites on average in view and the satellite geometry is poor (Figure 9). As we applied a Kalman filter to kinematic PPP, the non-nominal yaw attitude also had impacts on the following epochs. Figure 10 shows the Root Mean Square (RMS) of three directions from the 597th epoch (the starting epoch of shadow crossing) to the 1200th epoch. This figure shows that the accuracy of strategy 3 is significantly better than that of strategies 1 and 2 .

4.2.2.2. Positioning error results from different satellite types during eclipsing periods. For the further analysis on the effects of non-nominal yaw attitude of 


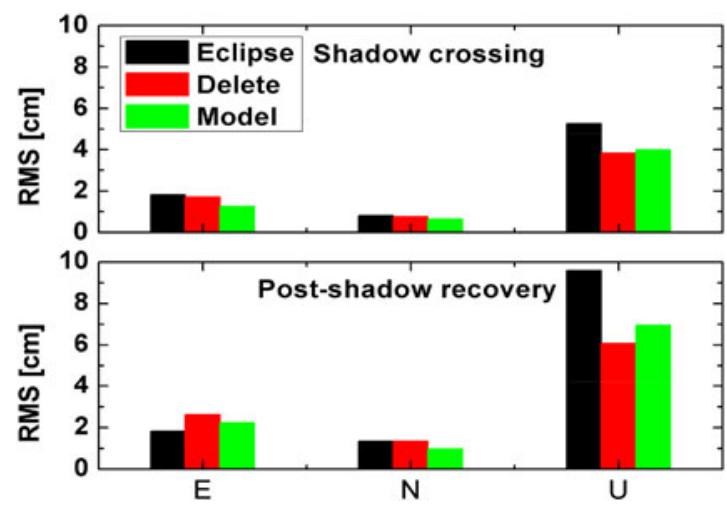

Figure 11. RMS of kinematic PPP based on three strategies during the period of non-nominal yaw attitude for BLOCK IIA (G26).

BLOCK IIA and BLOCK IIF on the kinematic PPP solutions (as periods during noon turn and shadow crossing for BLOCK IIR are too short, we do not analyse these periods here), in the following tests we selected the data where only one satellite is in an eclipsing period and calculated the statistics of positioning accuracy for the eclipsing periods. Figure 11 shows the RMS of 21 arcs of non-nominal yaw attitude (BLOCK IIA G26) for stations ALGO, GODE and AMC2 from DOY 344 to DOY 350 of 2012. These results show the accuracy in the East and North directions for strategy three (Model) which was 20\% 30\% better than those by strategy 1 (Eclipse) and strategy 2 (Delete). The accuracy in the Up direction is improved by $30 \%$ for strategy 3 compared with strategy 1 and is equal to the accuracy for strategy 2. During the period of post-shadow recovery, the positioning accuracy for the three strategies is worse than that in shadow crossing, and the accuracy for strategies 2 (Delete) and 3 (Model) in the direction of North and Up are better than that for strategy 1 (Eclipse). However, in the East direction, the accuracy for strategy 2 and 3 is worse than strategy 1 (Eclipse), and this is mainly because of the $2 \mathrm{~cm}$ bias caused by strategies 2 and 3 . The statistical value is $1 \cdot 14 \mathrm{~cm}$ after this bias is removed.

Figure 12 shows the RMS of 21 arcs of non-nominal yaw attitude (BLOCK IIF G01) for stations ALGO, CONZ and AMC2 from DOY 78 to DOY 84 of 2012. It shows that strategy 3 (Model) can slightly improve the positioning accuracy, but the differences in positioning accuracy by the three strategies are insignificant.

From Figures 11 and 12, we can see that the non-nominal yaw attitudes of BLOCK IIA satellites mainly affect the Up direction, especially during the shadow-recovery periods, and the non-nominal yaw attitude of BLOCK IIR and BLOCK IIF has little impact on the positioning error, and thus users can even ignore the impact of non-nominal yaw attitudes of the BLOCK IIR and BLOCK IIF. To further investigate the mitigating strategy including deleting the measurements of non-nominal yaw attitude and using the yaw-attitude model, we compare the kinematic PPP solutions of 15 IGS stations (Figure 2).

We used the coordinates of the IGS stations from the IGS SINEX files as ground truth and calculated the positioning RMS, and then calculated the positioning RMS 


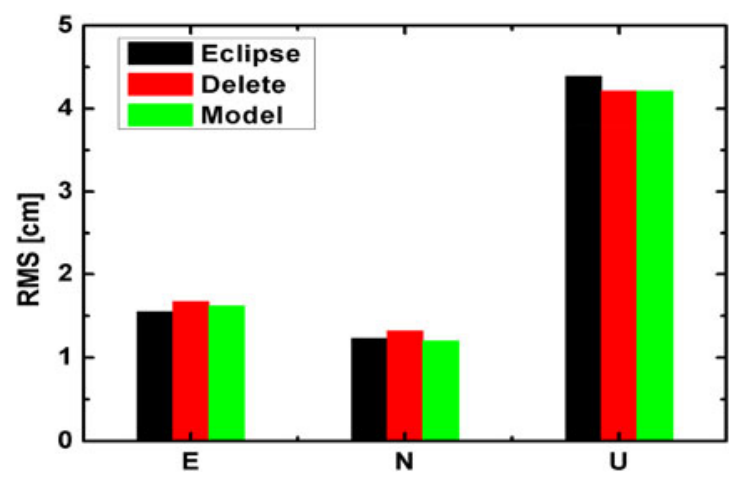

Figure 12. RMS of kinematic PPP based on three strategies during shadow crossing for BLOCK IIF (G01).

differences of the strategy 2 (Delete) and strategy 3 (Model) with respect to strategy 1 (Eclipse) for each day. From the daily results of the RMS differences for all the stations, we can see that the impact of non-nominal attitudes on kinematic PPP is different for different stations. Figure 13 presents the daily RMS differences of three strategies at station ARTU and BRFT. It is seen that the impact of non-nominal yaw attitude is different at the two stations, the RMS of the Up direction for some days is significantly improved either by modelling the non-nominal attitude or deleting the data during the eclipsing period. Figure 13 also shows that strategy 2 (Delete) and strategy 3 (Model) cannot always improve the positioning accuracy, so we make statistics of the days with an improvement and calculate the ratio of improved days to total days for the selected stations respectively (Figure 14). We can see about $80 \%$ of days in the horizontal and $75 \%$ in the Up direction with an improvement and the comparison of strategy 2 (Delete) and 3 (Model) shows that strategy 3 is slightly better than strategy 2 in accuracy improvement. Considering the impacts of different satellite types, we also compared RMS improvement under different conditions, as shown in Figure 15. About $60 \%$ of the RMS improvement is from 0 to $10 \mathrm{~cm}$ for all conditions and the RMS improvement for the days where only the BLOCK IIR and IIF satellites have nonnominal attitude is larger than that for the days where the BLOCK II/IIA satellites are in the eclipsing periods. This is because the effects of non-nominal attitudes for BLOCK IIR and IIF satellites on the PPP solution are less than that for BLOCK II/IIA satellites and the eclipsing period is short for most of the time during one day. On the other hand, it is clearly shown that strategy 3 (Model) is about 10\% better than strategy 2 (Delete). As compared to strategy 1 (Eclipse), the other two strategies have worse accuracy on some days. However, this is normal because the model cannot completely eliminate the error caused by the non-nominal attitude and similarly deleting the data during eclipsing period is not always favourable, as shown in Figure 9.

5. CONCLUSIONS AND SUGGESTIONS. Based on the comparison between the nominal and the modelled yaw angle, this paper analysed the impact of nonnominal attitudes on different GPS satellite types in eclipsing seasons. During the 

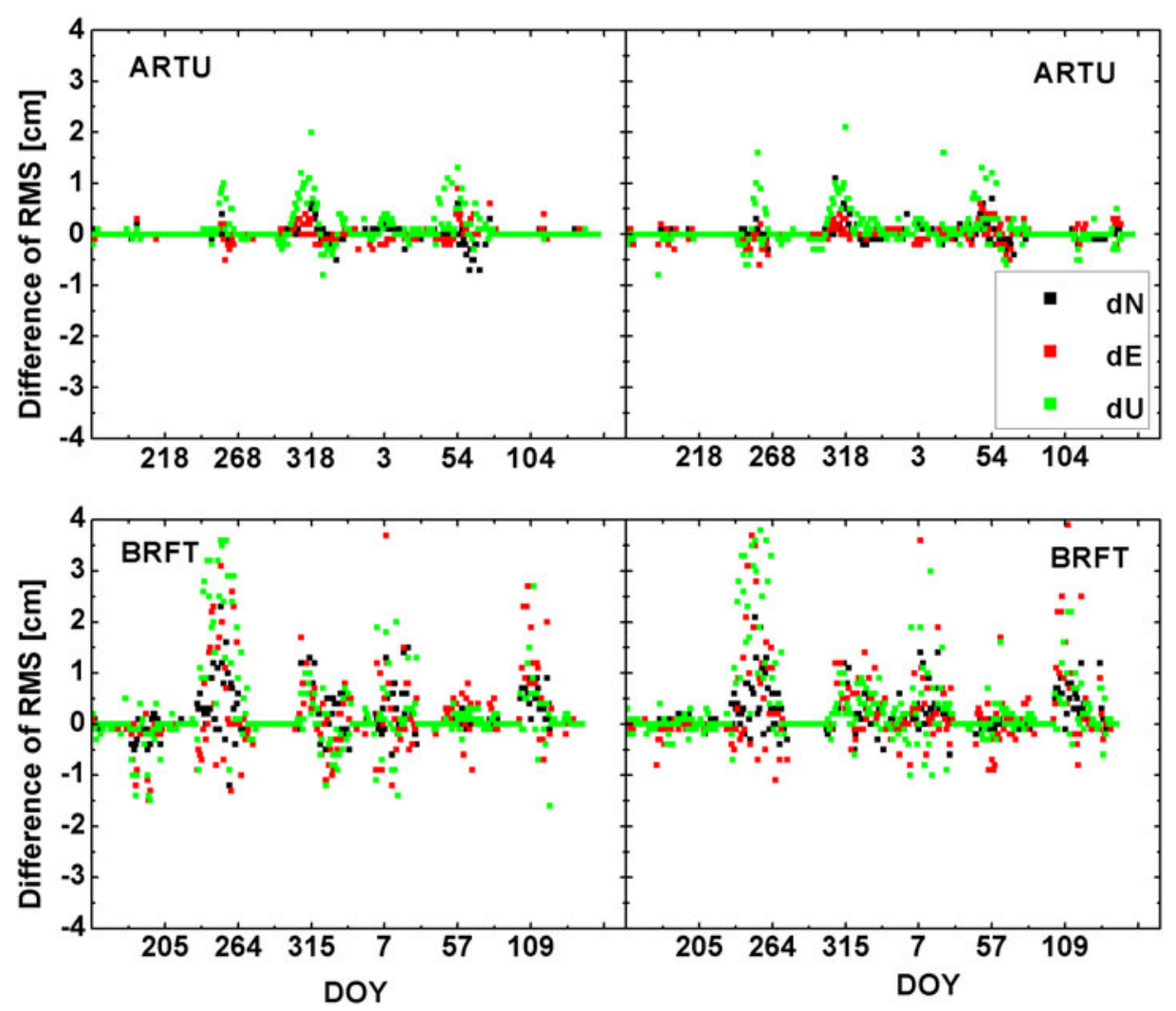

Figure 13. Daily RMS differences of the three strategies. The left column is the difference between strategy 3 (Model) and strategy 1 (Eclipse), and the right column is the difference between strategy 3 (Delete) and strategy 1 (Eclipse).

noon turn periods, the differences between nominal and modelled yaw angle of different satellite types are all related to the Sun elevation angle in a similar regime. During the shadow crossing periods, the regime varies for different satellite types. The difference between the nominal and the modelled yaw angle of the BLOCK II/ IIA satellite is the largest, while the difference for BLOCK IIF is small, except for the periods when the Sun elevation angle is close to $0^{\circ}$. The post-shadow recovery periods for BLOCK IIF satellites are very short while the periods for BLOCK II/ IIA are much longer.

By applying precise ephemeris from different $\mathrm{ACs}$ as well as products generated with PANDA software based on different yaw attitude models, the performance of PPP is evaluated in terms of both positioning accuracy and residuals distribution for a large data set. It is demonstrated that high accuracy positioning is also achievable once the yaw-attitude model keeps consistent on both "server" and "user" sides. The experimental results of kinematic PPP demonstrate that the non-nominal yaw attitude of the BLOCK II/IIA satellites has the greatest impact on PPP solutions with a typical bias of $20 \sim 30 \mathrm{~cm}$ during the shadow crossing. By applying the yaw-attitude model, its accuracy can be improved by $20 \% \sim 30 \%$. In contrast, the influence of 


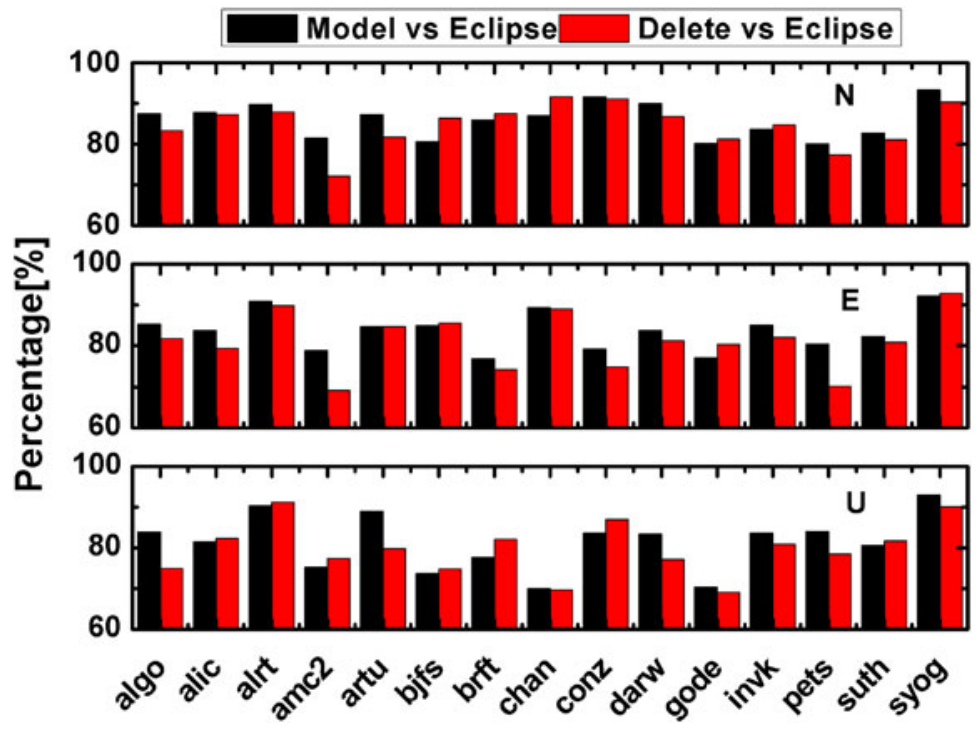

Figure 14. The ratio of improved days to total days for each IGS stations using different strategies.

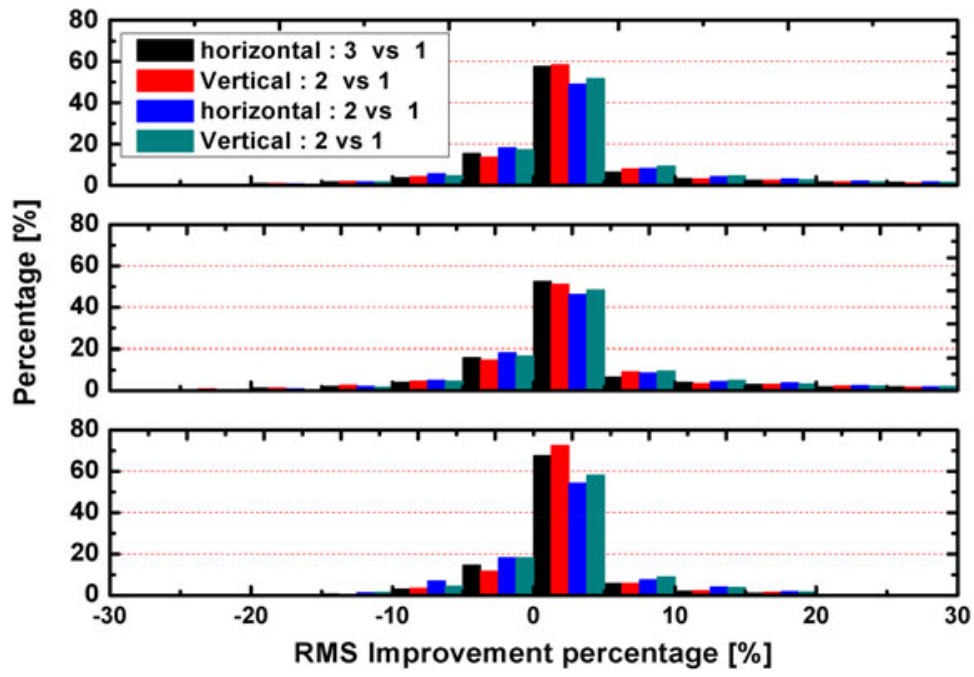

Figure 15. The histogram for the RMS improvements when using different strategies. Note that the improvements larger than $30 \%$ are excluded. The number 1 to 3 represents different strategies (Eclipse, Delete and Model respectively). The top panel is the statistic for all the days with nonnominal attitude satellite, the middle panel is the statistic for days with only BLOCK II/IIA satellites eclipsed and the bottom panel is the statistics for the days that the BLOCK IIR and BLOCK IIF satellites have non-nominal attitudes.

non-nominal yaw attitudes of the BLOCK IIR and BLOCK IIF satellites on the positioning is not so significant. Generally, by deleting the measurement of non-nominal yaw attitude, PPP users can also achieve a considerable positioning accuracy, however, when the satellite geometry is relative poor, the positioning accuracy applying 
the yaw attitude model is $30 \%$ better than the result with the measurement of nonnominal yaw attitude deleted.

In summary, it is suggested that PPP users should apply the corresponding attitude model to that used in orbit and clock products. Furthermore, in order to achieve suitable positioning accuracy under an unfavourable environment with poor satellite geometry, users should apply the yaw attitude model rather than delete the observations of non-nominal yaw attitude.

\section{REFERENCES}

Bar-Sever, Y.E. (1996). A new model for GPS yaw-attitude. Journal of Geodesy, 70, 714-723.

Bisnath, S. and Gao, Y. (2008). Current state of precise point positioning and future prospects and limitations. Observing our Changing Earth, 133, 615-623.

Dilssner, F. (2010). GPS IIF-1 satellite - antenna phase centre and attitude modelling. InsideGNSS, Sept 2010, 59-64.

Dilssner, F., Springer, T. and Enderle, W. (2011). GPS IIF yaw attitude control during eclipse season. In AGU Fall Meeting Abstracts.

Dow, J.M., Neilan, R.E. and Rizos, C. (2009). The international GNSS service in a changing landscape of global navigation satellite systems. Journal of Geodesy, 83(3-4), 191-198.

Geng, J., Teferle, F.N., Meng, X. and Dodson, A.H. (2010). Kinematic precise point positioning at remote marine platforms. GPS Solutions, 14(4), 343-350.

Geng, J., Teferle, F.N., Meng, X. and Dodson, A.H. (2011). Towards PPP-RTK: Ambiguity resolution in real-time precise point positioning. Advanced Space Research, 47(10), 1664-1673.

Kouba, J. and Heroux, P. (2001). Precise point positioning using IGS orbit and clock products. GPS Solutions, 5(2), 12-28.

Kouba, J. (2009a). A guide to using International GNSS Service (IGS) products. International GNSS.

Kouba, J. (2009b). A simplified yaw-attitude model for eclipsing GPS satellites. GPS Solutions, 13, 1-12.

Liu, J. and Ge, M. (2003). PANDA software and its preliminary result of positioning and orbit determination. Wuhan University Journal of Natural Science, 8(2), 603-609.

Niell, A.E. (1996). Global mapping functions for the atmosphere delay at radio wavelengths. Journal of Geophysical Research: Solid Earth, 101(B1), 3227-3246.

Saastamoinen, J. (1971). Atomospheric correction for the troposphere and stratosphere in radio ranging of satellites, in The Use of Artificial for Geodesy, Geophysical Monograph Series, 15, edited by S. W. Henriksen et al., 247-251, AGU, Washington, D.C.

Shi, C., Zhao, Q., Geng, J., Lou, Y., Ge, M. and Liu, J. (2008). Recent development of PANDA software in GNSS data processing. Proc. SPIE 7285, International Conference on Earth Observation Data Processing and Analysis (ICEODPA), 72851S. doi:10.1117/12.816261.

Wu, J.T., Wu, S.C., Hajj, G.A., Bertiger, W.I. and Lichten, S.M. (1993). Effects of antenna orientation on GPS carrier phase. Manuscripta Geodaetica, 18, 91-98.

Zhang, B., Ou, J., Yuan, Y. and Zhong, S. (2010). Yaw attitude of eclipsing GPS satellites and its impact on solutions from precise point positioning. Chinese Science Bulletin, 55(32), 3687-3693.

Zumberge, J.F., Heflin, M.B., Jefferson, D.C., Watkins, M.M. and Webb, F.H. (1997). Precise point positioning for the efficient and robust analysis of GPS data from large networks. Journal of Geophysical Research, 102(B3), 5005-5017. 